\title{
Verblisterte Arzneimittel
}

\section{Therapietreue leicht gemacht}

VoN R. DüsING

\begin{abstract}
Die patientenindividuelle Verblisterung von Arzneimitteln hilft vor allem chronisch kranken und älteren Patienten mit umfangreichen und komplexen medikamentösen Therapien, die ärztlichen Verordnungen einzuhalten.
\end{abstract}

- Ambulante medikamentöse Therapie ist dadurch gekennzeichnet, dass ein Patient sein Rezept einer Apotheke vorlegt und dafür das verordnete Medikament in der vom Hersteller abgepackten Einheit erhält. Der Patient entnimmt das Medikament dieser Verpackung in der verordneten Menge zu den festgelegten Einnahmezeitpunkten.

Dieses einfache Prinzip stößt an seine Grenzen, wenn die Therapie bezüglich Dosierung und Einnahmezeitpunkten komplexer und vor allen Dingen umfangreicher wird und wenn die betroffenen Patienten z. B. altersbedingt in ihrem Verständnis des Therapieschemas und entsprechend in ihrer Kooperation eingeschränkt sind. Therapieadhärenz, also das Umsetzen der ärztlichen Verordnung, ist unter solchen Bedingungen dann nicht mehr gewährleistet.

\section{Folgen mangelnder Therapietreue}

In Abhängigkeit von der zugrunde liegenden Erkrankung, dem Schweregrad des Krankheitsbildes (z. B. eine Herzinsuffizienz in den klinischen Stadien NYHA III-IV) und der Wirkdauer der einzelnen Wirkstoffe resultiert diese Konstellation dann in vermeidbarer Morbidität und Mortalität.

Auch die ökonomischen Folgen mangelnder Therapieadhärenz sind bedeutend. Durch unregelmäßige Me-

\footnotetext{
- Die Arbeit wurde unterstützt von assist Pharma GmbH, Merzig/Saarland.
}

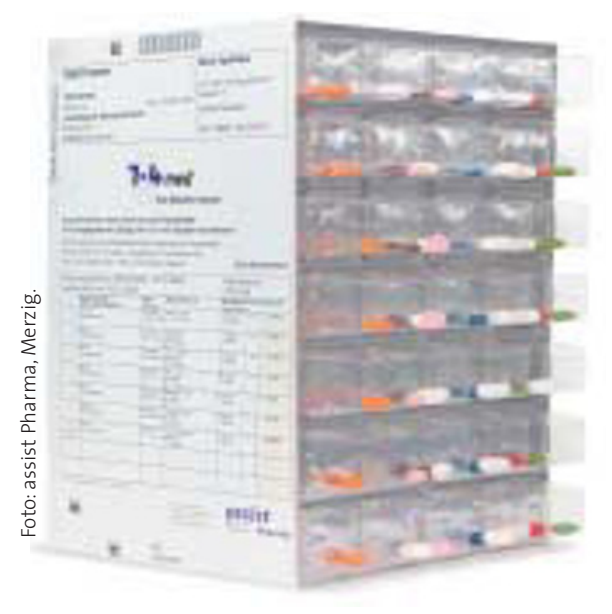

Der Wochenblister bietet für sieben Tage die Medikation in vier Tagesportionen an (morgens, mittags, abends, zur Nacht).

dikamenteneinnahme begünstigte oder ausgelöste akute Verschlechterungen des klinischen Zustands führen zu ambulanten Arztbesuchen, weiterführender Diagnostik, Umstellung der Therapie (Nichtadhärenz wird häufig als Nichtansprechen auf eine Therapie gedeutet) und - vermeidbarer - stationärer Krankenhausbehandlung.

\section{Patientenindividuelle Verpackung}

Eine Alternative zur klassischen Medikamentenverordnung ist die Abgabe von Arzneimitteln in patientenindividuellen Blisterpackungen (Sicht- und/ oder Durchdrückverpackungen). Die Arzneimittel werden dabei individuell für den jeweiligen Patienten nach Art, Dosis und Einnahmezeitpunkt sortiert und - in der Regel in Wochenblister abgepackt.

Bereits heute packen einige Apotheken auf Wunsch des Patienten (oder der Angehörigen oder des verordnenden Arztes) Arzneimittel in Wochenblister um. Dies ist ein zeit- und personalintensives Verfahren, das die Apotheken als individuelle Dienstleistung in der Regel kostenfrei wenigen ausgewählten Patienten anbieten können.
In Krankenhäusern sowie vielen Pflege- und Altenheimen kommt eine der Verblisterung vergleichbare Strategie zum Einsatz. Auch hier werden die Medikamente für die Mehrzahl der Patienten aus ihren Originalverpackungen entnommen und in einem TagesContainer dargereicht, wobei die einzelnen Dosierungszeitpunkte (morgens, mittags, abends, zur Nacht) unterschieden werden. In der Versorgung nicht stationärer älterer oder dementer Patienten übernehmen ambulante Pflegedienste oder betreuende Angehörige die Umverpackung. Eine Reihe von Tages- und Wochen-Containern sind hierzu im Handel verfügbar.

\section{Industrielle Verblisterung}

Entsprechend einer Novelle des Gesetzes über den Verkehr mit Arzneimitteln (AMG) ist seit 2005 eine Neuverblisterung von Arzneimitteln auch aus rechtlicher Sicht industriell möglich, da der Blister als Fertigarzneimittel definiert wird und eine Zulassungspflicht nicht gegeben ist. In einem derzeit im Saarland laufenden Pilotprojekt werden erste Erfahrungen mit diesem neuen Verfahren gesammelt, das voraussichtlich ab Spätherbst 2007 alternativ zur klassischen Medikamenten-Distribution zur Verfügung steht.

Literatur siehe Langfassung

Anschrift des Verfassers:

Prof. Dr. med. Rainer Düsing Universitätsklinikum Bonn

Medizinische Poliklinik

Wilhelmstr. 35-37

D-53111 Bonn

E-Mail:duesing@uni-bonn.de

Die ausführliche Langfassung ist im Originalienergänzungsband der MMW Nr. I/2007 erschienen. 